\title{
QUALIDADE DE SEMENTES DE FREIJÃO MACASSAR (VIGNA UNGUICULATA) EM RELAÇÃO Ã SANIDADE E GERMINAÇÃO
}

S. T. Barros - M. J. S. Fernandez Departamento de Micología Centro de Ciéncias Biologicas Universidade Federal de Pernambuco. Av. Prof. Artur de Sá s/n Cidade Universitária - 50739, Recife-Pernambuco-Brasil

M. Menezes

Departamento de Agronomia. Area de Fitossanidade Universidade Federal Rural de Pernambuco. Av. D. Manoel Medeiros s/n Dois Irmâos - 52071

Recife-Pernambuco-Brasil

Palabras clave: Semillas de frijol, germinación, hongos.

Key words: Cowpea seed, germination, fungi

\section{RESUMEN}

[Calidad de semillas de frijoles "Macassar" (Vigna unguiculata) en relación a aspectos sanitarios y de germinación.]

Veintidos variedades de semillas de frijol "macassar", Vigna unguiculata (L.) Walp, fueron examinadas con el objeto de verificar la población fúngica a ellas asociadas y su influencia en la germinación. Las semillas se recolectaron en 8 municipios siendo, el de Sáo José de Belmonte el que presento el mayor indice de hongos, siguiéndole Bezerros, Ibinajuba y Riacho-das-Almas.

Entre los hongos identificados se destacan Fusarium semitectum (26,9\%), Aspergillus niger $(16,7 \%)$, Fusarium oxysporum $(15,6 \%)$, Botryodiplodia theobromae $(11,1 \%)$ y Papulaspora $s p$, $(11,1 \%)$.

F. semitectum y F. oxysporum ocumieron con mayor frecuencia en el municipio de Riacho-dasAlmas, en los cultivos Pardo Mulatinho, Ramador, Branquinho. Las semillas que contenian estos organismos, presentaron podredumbre y por lo tanto no germinaron.

Botryodiplodia theobromae $y$ Papulaspora sp., fueron más comunes en los municipios de Bezerros y Sao José de Belmonte respectivamente. Estas especies ya fueron reportados por varios autores en semiWhas de frijol macassar, frijol común y bulbos de gladiolos, presentandose en todos ellos sintomas de podredumbre.

A. niger fué frecuente en los municipios de Bezemos y Triunfo, aunque no se considera patógeno de semillas, puede reducir la calidad de las mismas, al ser almacenadas, perjudicando su germinación.

\section{SUMMARY}

[Quality of cowpea seeds (Vigna unguiculata) in relation to sanitary conditions and germination.]

Seed samples of 22 cowpea Vigna unguiculata (L.) Walp. cultivars were bioassayed in order to obsenve the seedborme fungi, and their effect on seed germination. Of the eight counties from which the seeds were obtained, Sao José do Belmonte showed the highest incidence of fungi, followed by Bezerros, Ibirajuba and Riacho-das-Almas. Among the identified fungi were included Fusarium semitectum $(26,9 \%)$, Aspergillus niger (16,7\%), Fusarium oxysporum $(15,6 \%)$, Botryodiplodia theobromae $(11,1 \%)$ and Papulaspora sp. (11,1\%).

Fusarium semitectum and F. oxysporum occured with higher frequence in Riacho-das-Almas on Pando Mulatinho, Ramador and Branquinho cultivars. These fungi are known as seed pathogens. Although pathogenicity tests were not performed the seeds with these organisms showed rot and no germination.

Botryodiplodia theobromae and Papulaspora sp., were more frequent in Bezerros and Sâo José do Belmonte respectively. these fungi have been reported in seed of cowpea and bean, and gladiolos coms, causing rot symptoms.

Aspergillus niger was frequently observed in seed samples from Bezerros and Triunfo. Although this organism is not pathogen of seed, it can lower the quality of them during storage, and be prejudicial to germination.

Mortierella polycephala and Absidia cylindrospora var. cylindrospora were found in cowpea seeds by first time, and they apparently did not affect the seed germination. 
Mortierella polycephala $y$ Absidia cylindrospora var. cylindrospora fueron detectados por primera vez en semillas de frijol "macassar", aunque no ejercen aparentemente ningún efecto sobre la germinación de las mismas.

De las variedades analizadas, Sempre-Verde (Bonito y Saire), Costela-de-vaca (Ibirajuba) y Moita (Triunfo) presentaron un 90 a un 100\% de germinación y buenas condiciones fitosanitarias.

El análisis de regresión lineal simple reveló una correlación entre el número total de colonias de hongos y la disminución de la germinación.

\section{INTRODUÇÃO}

O feijáo macassar, Vigna unguiculata (L.) Walp. E uma cultura de grande importáncia para a regiáo Nordeste do Brasil, por ser um producto básico na alimentaçâo da populaçâo de baixa renda. Porém esta cultura está sujeita a perdas significativas de produçáo devido a ocorréncia de vàrias doenças, dentre elas encontram-se aquelas cujos agentes causais sâo comprovadamente transmitidos por sementes, prejudicando a cultura. Segundo Filgueiras (1981), o vigor da plântula està relacionado com a produtividade da cultura. Portanto, a presença de microrganismos nas sementes pode afetar a germinaçâo, emergência, vigor da plântula e, consequentemente, a produçâo. Menten (1978) tambén observou que o vigor das plântulas pode ser influenciado pela incidência de microrganismos nas sementes. Devido a importância da cultura do feijào, vàrios pesquisadores vém procurando estudar, qualitativa e quantitativamente, a ocorrência de fungos associados às sementes (Barros \& Menezes, 1981; Oliveira \& Bolkan, 1981; Choudhury, 1982; Menten, 1982; Barros et al., 1985), objetivando na maioria dos casos, a adoçâo de medidas adequadas de controle àquelas prejudiciais à qualidade das sementes.

$O$ presente trabalho teve como objetivo principal realizar um levantamento da populaçâo fúngica associada às sementes de feijâo macassar e observar possível influéncia dos microrganismos detectados, na sua germinaçâo.

\section{MATERIAL E METODOS}

Foram estudadas 22 cultivares de feijâo
Among the analysed cultivars, Sempre-Verde from Bonito and Saire counties, Costela-de-Vaca from Ibirajuba and Moita from Triunfo showed the seed germination of $90-100 \%$, and good phytosanitary conditions

Nevertheless, there was a significant positive correlation between total number of fungi and decrease of gemination.

macassar procedentes de oito municipios do Estado de Pernambuco-Brasil, assim distribuídas: Riacho das Almas (Olho-de-Pombo, Moita, Ramador e Pardo Mualtinho); Belo Jardim (SempreVerde e Quarentinha); Bezerros (Manteiga, Boca Preta, Sempre-Verde e Calaniâo); Saire (SempreVerde), Sâo José do Belmonte (Chico Modesto, Canapú, Seridó e Feijâo Comum); Ibirajuba (Costela-de-Vaca, Rabo-de Tatú e Rasga Letra), Bonito (Sempre-Verde) e Triunfo (Moita e Quarentinha) com objetivo de se observar a sanidade das sementes, no que tange a populaçâo fúngica $\mathrm{e}$ sua inflúencia na germinaçâo.

Em condiçoes assépticas, as sementes foram desinfestadas durante 2 minutos, em soluçâo de hipoclorito de sodio a $1,5 \%$. Após esse tratamento, as sementes foram lavadas duas vezes em água esterilizada e, em seguida plaqueadas, utilizando-se o método de papel de filtro. Foram analisadas 100 sementes de cada cultivar (10 por placa de Petri) incubadas a temperatura ambiente durante 8 dias. Após este período foi efetuada a avaliaçâo, identificando-se os fungos desenvolvidos sobre as sementes, com base principalmente nas características morfológicas dos conídios e outras estruturas consideradas importantes para determinaçâo de géneros e espécies, seguindo-se em alguns casos a literatura disponível (Snyder \& Hansen, 1940; Thom \& Raper, 1945; Raper \& Thom, 1949; Gilman, 1963; Messiaen \& Cassini, 1968; Barnett \& Hanter, 1972; Ainsworth et al. 1973; Alexopoulos, 1977; Webster, 1978; Sutton, 1980; Domsch et al. 1980). A percentagem total dos fungos presentes nas cultivares, a percentagem de sementes germinadas por cultivar, e a dismuiçâo da germinaçâo, foram determinados aplicando analises de regressâo linear simples. 


\section{RESULTADOS E DISCUSSÃo}

As espécies de fungos encontradas nas sementes de feijâo macassar, somaran um total de 461 colonias, havendo fungos mais frequentes que outros, conforme mostrado na Tabela 1.

Dos oito municípios que foram coletadas as cultivares de feijâo macassar o de Sâo José do Belmonte foi que apresentou-se com maior índice de assinalamento de fungos, seguindo-se Bezerros, Ibirajuba e Riacho-das Almas. Dos fungos mais frequentes no município de Sâo José do Belmonte, destacou-se Papulaspora sp. que foi detectado na cultivar Chico Modesto, a qual mostrouse mais sujeita a infecçâo por fungos patógenos e de armazenamento. Este organismo foi detectado nas superficies das sementes apodrecendo-as. Barros et al (1985) tambén ja relataram Papulaspora sp, em sementes de feijâo macassar, as quais nâo germinaram. Hotson (1942) ja havia isolado algumas espécies de Papulaspora de bulbos de gladiolos que apresentavam sintomas de apodrecimento.

Fusarium semitectum e Fusarium oxysporum foram detectados com maior frequência no municipio de Riacho-das-Almas, nas cultivares Pardo Mulatinnho, Ramador e Branquinho. Com relaçâo a F. semitectum, este organismo foi detectado por Barros et al (1985) em feijâo macassar mostrandose patogénico às sementes. Dhingra et al (1978) revelaram que $\mathbf{F}$. semitectum penetra através da parede das vagens infectando as sementes e prejudicando a germinaçâo.

F. oxysporum foi encontrado com certa frequência em outras amostras de feijâo macassar no Estado de Pernambuco, por Barros et al. (1985). Apesar de nâo ter sido realizado teste de patogenicidade, esta espécie causou apodrecimento das sementes. Tambén Oliveira \& Bolkan (1981) isolaram F. oxysporum de sementes de feijâo procedentes de vários Estados do Brasil, tendo este fungo se mostrado patogénico quando inoculado nas cultivares Branquinho, Canapu, Sempre-Verde e Pitíba, tanto "in vitro" como em casa-devegetaçâo. Do mesmo modo, Barros (1981) verificou a patogenicidade deste fungo, oriundo de sementes de feijâo macassar, quando inoculado nas cultivares Alagoano e Quarenta Días

Botryodiplodia theobromae foi detectado nos municipiós de Ibirajuba, Bezerros, Sâo José do Belmonte e Riacho-das Almas, sendo registrado com mais frequência no município de Bezerros, na cultivar Calaniâo, cujas sementes apresentaram sintomas de apodrecimento. Este fungo já foi reportado por vários pesquisadores como Lasca (1987); Menezes et al. (1981); Oliveira \& Bolkan
(1981) e Barros \& Menezes (1981) em sementes de feijâo comum e macassar, prejudicando a germinaçâo.

Phomopsis sp. apesar de ter sido detectado com baixa frequência nos municípios de Belo Jardim, Ibirajuba, Sâo José do Belmonte, Bezerros, Triunfo e Riacho-das-Almas, causou podridâo nas sementes, e consequentemente prejudicou a germinaçâo. Maia et al. (1990) estudiaram sua patogenicidade em condiçôes de casa-de-vegetaçâo e verificaram que as sementes germinaram normalmente, ocorrendo no entanto necroses e deformaçôes foliares. Também Oliveira \& Bolkan (1981) relataram a patogenicidade de Phomopsis sp. quando inoculado nas cultivares Branquinho, Canapu, SempreVerde e Pitiuba.

Aspergillus niger e Rhizopus oryzae foram encontrados apenas colonizando o tegumento das sementes, sem causar problemas de podridâo. Estes organismos embora nâo sejam considerados patógenos de sementes podem, no entanto, reduzir a qualidade das mesmas quando armazenadas, prejudicando posteriormente o "stand" de germinaçâo.

Macrophomina phaseolina, importante patógeno de feijâo macassar no Estado de Pernambuco, foi detectado com baixa freqüência no município de Sâo José do Belmonte. Porém, Barros et al. (1985) detectaram este fungo com alta freqüência nas cultivares procedentes dos municípios de Araripina, Serra Talhada e Limoeiro em Pernambuco. As sementes que estavam colonizadas, apresentaram podridâo. De acordo com Kendrick, citado por Muniz \& Muchovej (1987), M. phaseolina penetra na plântula através da base do nódulo cotiledonar, especialmente em sementes que estâo internamente infectadas. Outros pesquisadores como Muniz \& Muchovej (1987), Yamashita \& Menezes (1988), Charchar et al, (1988); Barros et al. (1988) e Oliveira \& Mello (1988) ressaltam a presença deste fungo em sementes de feijâo comum, ervas daninhas, trigo, feijâo macassar e amendoim.

No presente trabalho, a analise dos dados revelou efeito significativo para a regressâo linear simples entre o total de colonias de fungos e decréscimo na germinaçâo, ocorrendo correlaçâo positiva $(r=0,8448)$, o que sugere que para cada $1 \%$ de colonia há uma díminuiçâo de igual valor na germinaçâo das sementes.

Os resultados mostraram maior percentagem de germinaçâo em relaçâo as cultivares Costela-deVaca $(100 \%)$, Sempre-Verde $(100 \%)$ e Moita (95\%), respectivamente dos municípios de Ibirajuba, Bonito e Triunfo. Por outro lado, a mais baixa percentagem de germinaçâo ocorreu em relaçâo âs cultivares Chico Modesto (28\%), procedente de Sâo José do Belmonte; Branquinho (28\%), Rama- 
dor (46\%) e Pardo Mulatinho (65\%), procedentes de Riacho-das-Almas; Calaniâo (54\%) e Manteiga $(60 \%)$ de Bezerros. Em general, as sementes das cultivares acima citadas apresentaram alguns fungos tais como Botryodiplodia theobromae, Papulaspora sp., Fusarium oxysporum, Fusarium semitectum e Macrophomina phaseolina, os quais causaram apodrecimento das sementes colonizadas, e, consequentemente reduziram a germinaçâo. Das cultivares analisadas $76,9 \%$ germinaram normalmente mesmo apresentando certos fungos em suas sementes, como Aspergillus tamarii, Aspergillus flavus, Aspergillus fumigatus, Aspergillus ochraceus, Aspergillus restrictus, Acremonium sp., Penicillium variabile. Estes fungos foram citados por alguns pesquisadores (Ito et al., 1982; Mentem. 1982; Aguiar et al., 1982 e Barros et al., 1985) em sementes de caupi, soja e sorgo. Segundo Neergaard (1970), certos microorganismos de sementes podem exercer influência somente durante 0 desenvolvimento das plántulas. Ellis et al. (1976) observaram que a diminuiçâo da germinaçâo pode estar associada a fungos presentes nas sementes, uma vez que, quando a percentagem de infecçâo aumenta, a percentagem de germinaçâo e emergencia no campo diminui.

Para as cultivares Sempre-Verde, Ramador e Moita foi possível a análise de amostras coletadas em diferentes municípios, permitindo assim observar que o grau de infecçâo das sementes pode variar com a resistencia da cultivar envolvida, e, tambćn com as condiçôes ambientais da regiâo produtora. De modo geral a cultivar Sempre-Verde mostrou melhor qualidade de suas sementes, em sanidade e germinaçâo, variando no entanto de acordo com a sua procedência. Assim, as sementes produzidas nos municípios de Bonito (0 colonia e $100 \%$ de germinaçâo) e Sairé ( 1 colônia e $100 \%$ de germinaçầo) apresentaram melhor qualidade em relaçâo aquelas produzidas em Belo Jardim (13 colônias e $93 \%$ de germinaçâo) e Bezerros (6 colônias e $86 \%$ de germinaçâo). A cultivar Ramador produzida em Belo Jardim apresentou-se menos infectada por fungos (14 colônias $89 \%$ de germinaçâo) do que a amostra coletada em Riacho-das-Almas ( 71 colônias e $47 \%$ de germinaçâo). Já a cultivar Moita produzida em Triunfo mostrou melhor qualidade de suas sementes ( 0 colônias e 95\% de germinaçâo) que áquela de Riacho-dasAlmas ( 6 colônias e $84 \%$ de germinaçâo). A influéncia do ambiente na qualidade das sementes ja foi ressaltada por Neergaard (1979), sendo a umidade um importante fator para o estabelecimento de microrganismo e que o estado de sanidade das semente pode variar de um ano para o outro de acordo com as condiçôes ambientais, incluindo-se principalmente, temperatura e umidade predominantes no ano de sua produçâo.

Dentre as espécies detectadas cita-se Mortierella polycephala, de ampla distribuiçâo geográfica, já tendo sido isolado de solos cultivados com canade-açúcar, nos Estados de Pernambuco e Rio Grande do Norte (Upadhyay, 1967) de raízes de alfafa (Moskovets, 1957), de plantas de jardim (Domsch, 1960), da rizosfera de cravo-da-india (Luganskas \& Grybanskiene, 1970) e da rizosfera do trigo, tomate e repolho (Khalabuda, 1973). Sendo um fungo do solo, e considerando-se os relatos de Neergaard (1970) sobre transmissibilidade de patógenos por sementes acredita-se que a presença de $\mathbf{M}$. polycephala no semente de feijâo macassar possa ser explicada pela sua penetraçao através do sistema radicular e translocaçâo pela força de transpiraçâo até os órgâos florais, permanecendo na semente sem causar dano aparente. $\mathrm{O}$ mesmo pode ser dito com relaçâo á especie Absidia cylindrospora var. cylindrospora, fungo tambén do solo, de ampla distribuiçâo geográfica, já tendo sido reportado por vários pesquisadores (Hagen, 1908; Nielsen, 1930; Picci \& Verona, 1956; Jensen, 1963; Hesseltine \& Ellis, 1964; Oener, 1970; Grochenaur, 1970). Pidoplichko et al. (1960) isolaram este organismo de raízes de gramíneas e Holubova-Jechova (1970) encontrou o fungo deteriorando castanha do Pará. Em semen. tes de feijâo este é o primeiro relato da ocorrencia dos fungos M. polycephala e A. cylindrospora var. cylindrospora e suas sementes.

\section{AGRADECIMIENTOS}

Os autores agradecem ao Prof. Dr. Gilvan Pio-Ribeiro do Departamento de Agronomia, Area Fitossanidade de Universidade Federal Rural de Pernambuco c á Sra. Maria Lúcia Rodrigues Olivares, pel cooperaçâo prestada a este trabalho. 
Tabela 1

Percentagem total de fungos associados às sementes de cultivares de feijâo macassar, procedentes de oito municípios do Estado de Pernambuco - Brasil

\begin{tabular}{|c|c|c|c|c|c|c|c|c|c|c|}
\hline MUNICIPIOS & 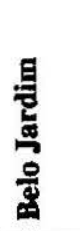 & 莡 & 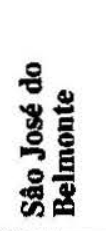 & 密 & 营 & 窇 & 巴巳 & 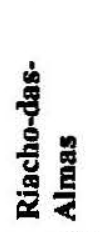 & 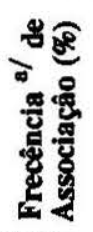 & 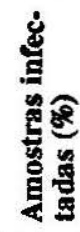 \\
\hline $\begin{array}{l}\text { Fusarium semitectum } \\
\text { Berk \& Rav. }\end{array}$ & 4,3 & - & 0,7 & 0,2 & - & - & 3,0 & 18,7 & 26,9 & $36 ; 4$ \\
\hline Aspergillus niger van Tieghem & - & - & 2,4 & 7,4 & - & - & 5,2 & 1,7 & 16,7 & 40,9 \\
\hline $\begin{array}{l}\text { Fusarium oxysporum Schlecht. } \\
\text { emend Snyd \& Hans. }\end{array}$ & - & 0,2 & 0,7 & 5,0 & - & - & - & 9,7 & 15,6 & 45,5 \\
\hline $\begin{array}{l}\text { Botryodiplodia theobromae (= Lasiod } \\
\text { theobromae (Pat.) Griff \& Maubl.) }\end{array}$ & liplod & 1,8 & 1,1 & 6,5 & - & - & - & 1,7 & 11,1 & 40,9 \\
\hline Papulaspora sp. & - & - & 10,8 & 0,3 & - & - & - & - & 11,1 & 18,2 \\
\hline Rhizopus oryzae Went \& Prins. Geer & 0,2 & - & 4,8 & - & - & - & 0,6 & - & 5,6 & 27,3 \\
\hline Phomopsis sp. & 1,5 & 0,4 & 1,5 & 1,2 & - & - & 0,4 & 0,4 & 5,4 & 45,5 \\
\hline Aspergillus oryzae (Ahlburg) Cohn & - & - & 0,7 & 1,0 & - & - & - & - & 1,7 & 18,2 \\
\hline Aspergillus tamarii Kita & - & 0,2 & 1,5 & - & - & - & - & - & 1,7 & 9,0 \\
\hline Aspergillus flavus Link ex Gray & - & 0,5 & 0,3 & - & - & - & - & 0,3 & 1,1 & 13,6 \\
\hline Aspergillus ochraceus Wilhelm & - & - & 1,1 & - & - & - & - & - & 1,1 & 13,6 \\
\hline Acremonium sp. & - & - & 0,4 & - & - & - & - & - & 0,4 & 4,5 \\
\hline Aspergillus restrictus G. Smith & - & - & 0,2 & - & - & - & - & - & 0,2 & 4,5 \\
\hline Aspergillus fumigatus Fres. & - & - & - & - & - & 0,2 & - & - & 0,2 & 4,5 \\
\hline $\begin{array}{l}\text { Absidia cylindrospora Hagem } \\
\text { var. cylindrospora }\end{array}$ & - & - & - & 0,2 & - & - & - & - & 0,2 & 4,5 \\
\hline Mortierella polycephala Coemans & - & - & 0,2 & - & - & - & - & - & 0,2 & 4,5 \\
\hline Penicillium variabile Sopp & - & 0,2 & - & - & - & - & - & - & 0,2 & 4,5 \\
\hline $\begin{array}{l}\text { Macrophomina phaseolina } \\
\text { (Tassi) Goid }\end{array}$ & - & - & 0,2 & - & - & - & - & - & 0,2 & 4,5 \\
\hline
\end{tabular}

1/ Percentagem para cada fungo foi determinada em relaçåo ao número total de colônias formadas. 


\section{REFERENCIAS}

I. AGUIAR, N.T.O.; BARROS. S.T.; FERNANDES, M.J.S.; AGLIAR, L. A. B. (1982). Populaçâo fúngica de sementes de sorgo, Sorghum bicolor (L.) Moench procedentes de quatro município do Estado de Pernambuco e toxigenidade de Aspergillus flavus isolados. Fitopal. Bras. 7: 471.

2. AINSWORTH, G.C.: SPARROW. F.K.: SUSSAN, A.S. (1973). The fungi: An Advanced Treatise. Academic Press. New York.

3. ALEXOPOULOS. C.J. (1977). Introduccion a la Micología. Editorial Universitária. Buenos Aires.

4. BAR NETT. H.I. \& HUNTER, B.B. (1972). Illustrated genera of imperfect fungi, Burgess Publishing, Minneapolis

5 BARROS. S.T. (1981). Fungos de sementes de feijâo macassar. Fditora Lniversitária. Recife.

6. BARROS, S.T. \& MENEZES, M. (1981). Fungos associados ás sementes de feijâo macassar, Vigna unguiculata (L.) Walp. procedentes do município de Caruaru. Estado de Pernambuco. Fitopal. Bras., 6: 269-75.

7 BARROS, S.T.: MENIEZES. M.: FERNANDES. M.J.S. \& LIRA. N. P. (1985). Fungos associados ás sementes de 34 cultivares de feijâo macassar, Vigna unguiculata (L.) Walp. no Fstado de Pernambuco. Fitopal. Bras., 10: 85-95.

8. BARROS. S.T.: FERNANDES, M.J.S. \& LIMA, D.M.M. (1988). Inventário fúngico de sementes de cultivares de feijâo macassar. Vigna unguiculata (L.) Walp. Fitopal. Bras. 13: 127.

9. CHARCHAR, M.J.A.:NASSER, I.C.B. \& GOMES, A.C. (1988). Fungos associados as sementes de feijâo e trigo produzidos nas áreas irrigadas do Distrito Federal. Fitopal. Bras.. 13: 110.

10. CHOLDRHURY. M.M. (1982). Microflora fúngica de sementes de caupi em Ouricuri. Pernambuco. Fitopal. Bras., 7: 465 .

11. DHINGRA, O.D.: SEDIYAMA, C.; CARRARO, I.M. \& REIS. M.S. (1979). Bahavior of four soybean cultivars to seed infecting fungi in delayed harvest. Fitopal. Bras., 3: $277-282$.

12. DOMSCH. K.H. (1980). Das Pilzspertrum liner bodenprobe. 1. Nachweis der homogenitaet. Arch. Mikrobiol. 35: 181-195
13. DOMSCH, K.H.; GAMS, W. \& ANDERSON, TrauteHeidi. (1980), Compendium of soil fungi. Academic Press. London.

14. ELLIS, M.A.: GALVEZ G.E. \& SINCLAIR J.B. (1976). Effect of pod contact with soil on fungal infection of dry bean seeds. Plant. Dis. Rep. 60: 974-6

15. FILGUEIRAS. T.S. (1981). Seed vigor and productivity. Pesq. Agrop. Bras. 16: 851-854.

16. GILMAN. J.C. (1963). Manual de los hongos del suelo, Editorial Continental. Mexico.

17. GOCIIENAUR, S.E. (1970). Soil microflora of Peru. Mypath. Mycol. Appl. 42: 259-272.

18. HAGEN. O. (1908). Untersuchugen ueber Norwegische mucorineen. 1 Vid. Selsk. Skrifter, Math-natur. K 1. 1-50

19. HESSELTINE, C.W. \& ELLIS, J.J. (1964). The genus Absidia. Gongronella and cylindrical spored species of Absidia. Mycol. 56: 568-602.

20. HOLUBOVA-JECHOVA. V. (1970). The internal rots of Brazil nuts. Ceska Mykol. 24: 207-214.

21. HOTSON, H.H. (1942). Some species of Papulaspora associated with rots of gladiolus bulbs. Mycol. 34: 390-399.

22. ITO. M.F.: SOAVE. J.: MIRANDA. M.A. \& PARADELA. O. (1982). Testes preliminares visando conhecer o efeito da dureza do tegumento da semente de soja na incidencia de fungos. Fitopal. Bras. 7: 472 .

23. JENSEN. V. (1963). Studies on the microflora of danish beech forest soils. 5. The microfungi. Zentbl. Bakt. Parasitkde. Abt.. 117: 167-179.

24. KHALABLDA. T.V. (1973) Griby roda Mortierella, Izvo Naura, Moskva.

25. LASCA. C. (1978). Estudos sobre a flora fúngica de sementes de feijâo Phaseolus vulgaris (L.) O. Biológico, XLIV, 125-134.

26. LLGANSKAS. A.J. \& GRYBANSKIENE. V.J. (1970). The proteolytic activity of micromycetes growing in the clover root zone the roots themselves. Trudy Akad. Nauk Litov. SSR. Ser. C. 2: 25-31. 
27. MENEZES, J.R,; MOHAN, S.K; BLANCHINI, A. \& SOUZA, G.Z (1981). Qualidade sanitária de sementes de feijấo (Phaseolus vulgaris L.) no Estado de Paraná. Fitopal. bras., 6: 497-508.

28. MENTEN, J.O. (1978). Sanidade, germinaçáo e vigor de sementes de feijâo (Phaseolus valgaris L.) Summa Phytopathol., 4: 105-110.

29. MENTEN, J.O. (1982). Microflora fúngica de sementes de caupi (Vigna unguiculata) no Estado do Pará, Brasil. Fitopal. Bras., 7: 470 .

30. MESSIAEN, C.M. \& CASSINI, R. (1968). Recherches sur les Fusarioses. IV. La Systómatique des Fusartum. Ann. Epiph., 19: 378-454.

31. MOSKOVETS, V.S. (1957). The fungal microflora of lucerne rhizosphere in the Southem Ukranian SSR, 3. The fungal flora of lucerne roots. Mykrobiol. ZH. 19: 16-21.

32. MUNIZ, M. de F.S. \& MUCHOVEJ, J.J. (1987). Fungi associated with dry bean (Phaseolus vulgaris) seed in Alagoas. Fitopal. Bras., 12: 373-76.

33. NEERGAARD, P. (1970). Seedbome disease and Indian Agriculture. Proc. Ind. Nat. Sci. 36: 222-237.

34. NEERGAARD, P. (1979), Seed Pathology. The Mac Mi'llan Press. London.

35. NIELSEN, N. (1930). Fungi isolatred from soil and from excrements of animals. Meddel. Groenland, 74: 1-8.

36. OENER, M. (1970). Soil microfungi of Turkey. Mycopath. Mico. Appl., 42: 81-87.
37. OLIVEIRA, M.ZA. \& BOLKAN, H.A. (1981). Microflora fúngica de sementes de 18 variedades de caupi cultivadas em vários Estados do Brasil. Fitopal. Bras., 6: 57 .

38. OLIVEIRA, M.ZA. MELLO, S.C.M. (1988). Fungos associados a grâos de amendoim no recincavo da Bahia. Fitopal. Bras., 12: 128.

39. PICCI, G. \& VERONA, P. (1956). Qualche ricerca biologica sui terreni dello stato di S. Paolo del Brasile. Boll. Ins. Siroth., 35: 157-179.

40. PIDOPLINCHKO, N.M.; MOSKOVETS, V.S. \& ZHDANOVA, N.M. (1960). The effect on the shoots of some fungi the maize thizosphere. Mykrobiol. ZH. 22: 1520.

41. RAPER, K.B. \& THOM, C. (1949). A manual of the Ponicillin. Williams and Wilkings, Baltimore.

42. SNYDER, W.C. \& HANSEN, H.N. (1940). The species concept in Fusarium. Am. J. Bot. 27: 64-7.

43. SUTTON, C.B. (1980). The Coelomycetes. Fungi imperfect with pycnidia, acervuli and stromata. Commonwealth Mycological Institute. Kew.

44. THOM, C. \& RAPER, K.B. (1945). A Manual of the Aspergilli. Williams \& Wilkings, Baltimore.

45. UPADHYAY, H.P. (1967). Soil from Northeast Brazil. 3. Phycomycetes. Mycopath. Mycol. Appl. 31: 49-62.

46. WEBSTER, J. (1978). Introduction to fungi. Cambridge University Press, London.

47. YAMASHITA, C. \& MENEZEZ, J.R (1988). Fungos associados as sementes de algumas ervas daninhas no Estado do Paraná. Fitpal. Bras., 13: 122 